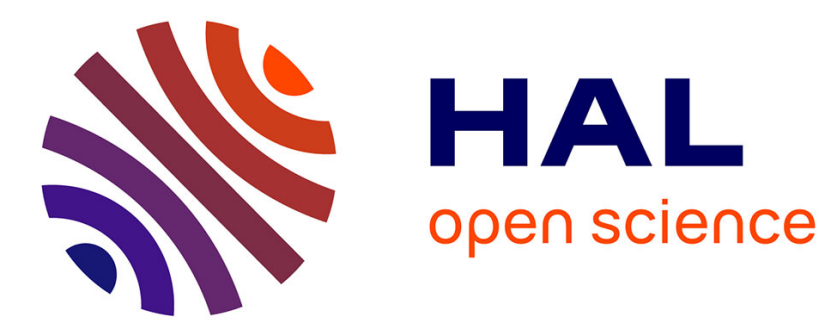

\title{
Expert opinion on managing chronic HCV infection in patients with type 2 diabetes mellitus
}

Luigi Elio Adinolfi, Ira M. Jacobson, Mark Bondin, Patrice Cacoub

\section{To cite this version:}

Luigi Elio Adinolfi, Ira M. Jacobson, Mark Bondin, Patrice Cacoub. Expert opinion on managing chronic HCV infection in patients with type 2 diabetes mellitus. Antiviral Therapy, 2018, 23 (Suppl 2), pp.11-21. 10.3851/IMP3255 . hal-01981560

\section{HAL Id: hal-01981560 https://hal.sorbonne-universite.fr/hal-01981560}

Submitted on 15 Jan 2019

HAL is a multi-disciplinary open access archive for the deposit and dissemination of scientific research documents, whether they are published or not. The documents may come from teaching and research institutions in France or abroad, or from public or private research centers.
L'archive ouverte pluridisciplinaire HAL, est destinée au dépôt et à la diffusion de documents scientifiques de niveau recherche, publiés ou non, émanant des établissements d'enseignement et de recherche français ou étrangers, des laboratoires publics ou privés. 


\title{
Beyond the Liver Spotlight Series: Type 2 Diabetes Mellitus
}

\section{Expert opinion on managing chronic HCV infection in patients with type 2 diabetes mellitus}

\author{
Luigi Elio Adinolfi, ${ }^{1}$ Ira Jacobson, ${ }^{2}$ Mark Bondin, ${ }^{3}$ Patrice Cacoub $b^{4-7^{*}}$ \\ ${ }^{1}$ Department of Medical, Surgical, Neurological, Metabolic, and Geriatric Sciences, Università \\ degli Studi della Campania "Luigi Vanvitelli", Naples, Italy ; ${ }^{2}$ NYU Langone Health, New York, NY, \\ USA; ${ }^{3}$ AbbVie, Inc. Chicago, IL, USA; ${ }^{4}$ Sorbonne Universités, UPMC Univ Paris 06, UMR 7211, and \\ Inflammation-Immunopathology-Biotherapy Department (DHU i2B), F-75005, Paris, France; \\ ${ }^{5}$ INSERM, UMR_S 959, F-75013, Paris, France; ${ }^{6}$ CNRS, FRE3632, F-75005, Paris, France; ${ }^{7}$ AP-HP, \\ Groupe Hospitalier Pitié-Salpêtrière, Department of Internal Medicine and Clinical Immunology, \\ F-75013, Paris, France, E-mail: patrice.cacoub@aphp.fr
}

*Corresponding author: Patrice Cacoub

\section{Abstract: 109 words \\ Main text: 4559 words \\ Number of display items: 5; 5 Figures and 0 Tables}

\section{Summary}

Type 2 diabetes mellitus (T2DM) has been identified as an extrahepatic manifestation of chronic HCV infection. Conversely, in the context of chronic HCV infection, T2DM can accelerate the course of HCVinduced liver disease leading to increased risk of fibrosis, cirrhosis, and hepatocellular carcinoma. The presence of T2DM negatively impacts the efficacy of interferon-based antiviral therapy, but real-world data with high-efficacy direct-acting antiviral therapies suggest high viral clearance rates in T2DM patients. In HCV-infected individuals, viral eradication is associated with a reduced risk of de novo T2DM in non-diabetic patients and beneficial metabolic changes in patients with T2DM, highlighting the importance of antiviral treatment and physician awareness of this association. 


\section{Introduction}

Hepatitis C virus (HCV) infection is a significant public health concern, with an estimated $1-2 \%$ of the global population with viraemic HCV [1,2]. Chronic HCV infection is a leading cause of liver-related morbidity and mortality, including cirrhosis of the liver, decompensated liver disease, hepatocellular carcinoma (HCC) and liver transplant [3]. In addition, chronic HCV infection is increasingly being viewed as a systemic disease with many extrahepatic manifestations [4]. Much of the data on extrahepatic manifestations of chronic HCV infection concern autoimmune and/or lymphoproliferative disorders, but HCV-associated cardiovascular, renal, central nervous system and metabolic disease have been reported [4]. Type 2 diabetes mellitus (T2DM) is a metabolic disorder characterized by insulin resistance (IR), increased hepatic glucose metabolism and a defect in insulin secretion. Insulin resistance and T2DM are more prevalent in chronic HCV-infected individuals versus those without HCV infection, and their presence may negatively affect disease progression $[5,6]$. An analysis of the strength of association between chronic HCV infection and extrahepatic manifestations classified IR and T2DM as Class B extrahepatic manifestations of HCV, defined as a higher prevalence in chronic HCV-infected patients compared with uninfected controls [4].

The objective of this paper is to review: the evidence for an association between T2DM and chronic HCV infection and the impact of T2DM on the clinical course of HCV infection; the proposed pathogenic mechanisms underlying the association between chronic HCV infection and T2DM; current recommendations for treatment of chronic HCV-infected patients with T2DM; and the impact of T2DM on HCV treatment, and the impact of HCV treatment on the incidence and clinical course of T2DM. 


\section{Association between T2DM and HCV infection}

Two meta-analyses found that chronic HCV infection was significantly associated with T2DM $[7,8]$. The more recent was a systematic review and meta-analysis of 102 studies published between 1996 and 2014 on extrahepatic manifestations of chronic HCV infection, and T2DM was found to be one of the most common extrahepatic conditions associated with chronic HCV infection [7]. This analysis identified 31 studies relating to HCV and T2DM, including 61,843 HCV-infected individuals and 202,130 uninfected controls. T2DM was present in approximately $15 \%(95 \% \mathrm{Cl}: 13 \%-18 \%)$ of patients with chronic HCV infection while $10 \%$ (95\% Cl: 6\%-15\%) of uninfected controls had T2DM, resulting in a pooled odds ratio (OR) for T2DM in HCV-infected patients of 1.58 (95\% Cl: 1.30-1.86) [7].

Multiple studies have shown an increased risk of T2DM in non-cirrhotic HCV-infected patients versus non-cirrhotic HBV-infected patients or the general population [9-11]. Additionally, those with HCVinduced cirrhosis or chronic liver disease have increased risk of T2DM compared with individuals with cirrhosis or chronic liver disease of non-viral origin $[8,12]$. Combined with evidence suggestive of HCV genotype-specific variation in T2DM prevalence between diabetic and non-diabetic individuals $[10,11]$ and in degree of IR [13], these data suggest a direct viral effect on the increased risk of T2DM, which is independent of cirrhosis or liver disease.

Another systemic review and meta-analysis evaluating, conversely, the risk of HCV infection in patients with T2DM likewise suggested a strong relationship between T2DM and HCV-infection. This analysis included 22 studies which included 12,426 individuals with T2DM and 65,625 controls (non-T2DM), and found that individuals with T2DM had 3.5-fold increased odds of having HCV infection compared to those without T2DM (OR = 3.50; 95\% Cl: 2.54-4.82) [14].

\section{What is the clinical relevance of T2DM in patients with chronic HCV?}


HCV-associated T2DM has been shown to accelerate the clinical course of chronic HCV infection, significantly increasing the risk of fibrosis and its progression to cirrhosis, decompensation and HCC [1524]. Multiple studies have shown a link between T2DM and the development of liver fibrosis. A metaanalysis on individual data from 3068 patients with chronic HCV GT1-4 infection in Europe, Australia and the United States found that those with fibrosis were more than twice as likely to have a history of diabetes as those without fibrosis (6.8\% versus $2.6 \%$, respectively; $P=0.0016)$ [15]. Similarly, an Italian study of 201 HCV GT1-infected patients found that patients with T2DM were twice as likely to have severe fibrosis (Scheuer score 3-4) as non-diabetic patients with IR (60\% versus 30\%, respectively; $P=0.006)[16]$. In this study, T2DM was significantly associated with severe fibrosis in multivariate analysis (OR: 2.7; 95\% Cl: 1.5-5.0; $P=0.001)$ [16]. In a study of Japanese HCV GT1- and GT2-infected patients, 33\% (32/97) of those with severe fibrosis had T2DM compared with only 5\% (4/74) of those with mild fibrosis $(P<0.001)$ [17]. An Italian retrospective analysis of patients with chronic HCV infection $(n=726)$ or chronic hepatitis B virus (HBV) infection $(n=126)$ found that T2DM was an independent risk factor for greater liver fibrosis (measured by Ishak staging) in HCV-infected patients $(P<0.05)$, but not in HBV-infected patients [18]. However, not all studies have reported an association between T2DM and advanced fibrosis. In contrast to the above results, an analysis of 1,461 chronic HCV-infected patients from the Swiss Hepatitis C Cohort Study with an estimated date of infection and at least one liver biopsy found no association between diabetes and accelerated fibrosis progression rate (defined as an increase of $\geq 0.13$ Metavir fibrosis units per year) [25]. In this study, diabetes was present in $8 \%(57 / 719)$ of patients without accelerated fibrosis progression and $9 \%(66 / 742)$ of those with accelerated fibrosis progression; univariate logistic regression analysis did not find an association between diabetes and accelerated fibrosis progression $(P=0.51)[25]$.

In a recent case-control study in chronic HCV-infected Chinese patients with or without cirrhosis, diabetes significantly increased the odds of developing cirrhosis (adjusted OR 2.1; 95\% Cl: 1.3-3.4; 
$P=0.001$ ), although the length of time the patient had diabetes, the type of diabetes treatment and the presence of complications of diabetes were not associated with development of cirrhosis [19]. Similarly, in a large Taiwanese cohort study of patients with chronic HCV infection ( $N=2132)$, diabetes was an independent risk factor for cirrhosis (adjusted HR: 2.5; 95\% Cl: 1.6-3.9; $P<0.001$ ) (Figure 1) and a larger effect was seen on the risk of developing decompensated cirrhosis (adjusted hazard ratio [HR]: 3.6; 95\% CI: $1.5-8.3 ; P=0.003)$ [20]. Additionally, in a retrospective study of 72,731 patients with compensated cirrhosis in the United States, those with a co-diagnosis of diabetes and cirrhosis were more likely to have a decompensation event than those with cirrhosis alone (OR: 1.14; 95\% Cl: $1.08-1.21 ; P<0.01)$ [21]. In two separate studies, diabetes was also shown to increase the risk of developing HCC in Chinese patients (adjusted OR 1.80; 95\% $\mathrm{Cl} 1.17-2.75 ; P=0.007$ ) [22] and Taiwanese patients (adjusted HR: 1.91; 95\% Cl: 1.10-3.30; $P=0.021$ ) [23] with chronic HCV infection. Additionally, in a large multinational cohort study of patients with chronic HCV infection and advanced fibrosis or cirrhosis (Ishak score 4-6; N=541), the 5-year cumulative incidence of HCC in patients with diabetes mellitus was $11.4 \%$ (95\% Cl: $3.0-19.8$ ) versus 5.0\% (95\% Cl: $2.2-7.8)$ in those without diabetes mellitus ( $P=0.013)$; in patients with an Ishak score of 6, diabetes mellitus was an independent risk factor for HCC (HR: 3.28; 95\% Cl: 1.35-7.97; $P=0.009$ ) (Figure 2) [24]. Further to these results, a single-centre, retrospective French study of 348 patients with HCV-associated cirrhosis found that diabetes mellitus was an independent predictor of death or liver transplant (HR: 1.34; 95\% $\mathrm{Cl}: 1.03-1.73 ; P=0.027)$, and was associated with major complications of cirrhosis, including renal dysfunction (HR: $2.35 ; 95 \% \mathrm{Cl}: 1.32-4.20 ; P=0.004$ ), bacterial infection (HR: 2.10; 95\% Cl: 1.23-3.59; $P=0.007)$ and HCC (HR: 1.94; 95\% Cl: 1.13-3.33; $P=0.016)[26]$. Independent of HCV infection, T2DM has been shown to increase the risk for acute myocardial infarction, stroke, lower-extremity amputation and end-stage renal disease (ESRD) [27], and is part of the cardiovascular disease continuum, culminating in end-stage heart disease [28]. It is not clear if the 
increased risk of cardiovascular disease in HCV infected individuals with T2DM is solely a consequence of IR or whether direct viral effects or systemic inflammation also contribute $[29,30]$.

\section{What are the proposed pathogenic mechanisms underlying the association between T2DM and chronic HCV infection?}

Induction of IR is thought to be the mechanism by which chronic HCV infection causes T2DM [29]. Chronic HCV infection is thought to lead to hepatic IR through direct interference with insulin signalling [29]. In humans, liver biopsy specimens taken from non-diabetic, HCV-infected patients showed inhibition of insulin-stimulated tyrosine phosphorylation of hepatic insulin receptor substrate-1 (IRS-1), leading to inhibition of the PI3-kinase/Akt insulin signalling pathway [31]. In a recent study using a mouse model of HCV-induced IR, HCV proteins directly impaired Glut2-mediated hepatic glucose intake and gluconeogenesis by downregulating IRS-2 and altering FoxO1 phosphorylation and nuclear exclusion insulin in hepatocytes [32]. In addition to HCV-induced hepatic IR, there is evidence of HCV-induced peripheral IR, predominantly in skeletal muscle [33,34]. Moreover, secretion of soluble endocrine mediators from infected hepatocytes may contribute to IR in extrahepatic tissues $[29,30]$. In a study of chronic HCV-infected patients with or without T2DM and healthy controls, chronic HCV infection was associated with IR and upregulation of tumor necrosis factor- $\alpha$, glucose- 6 -phosphatase and resistin, leading to gluconeogenesis [35]. Development of HCV-induced IR may be influenced by host-related genetic and environmental factors, and with additional contributions from an imbalance of adipocytokine profile and the presence of liver steatosis, may result in progression to T2DM [36].

In addition to IR, pancreatic $\beta$-cell dysfunction contributes to the development and progression of T2DM [37] and both IR and $\beta$-cell dysfunction have been demonstrated to contribute to glucose intolerance in HCV-infected patients [38]. Additionally, HCV has been observed in human pancreatic $\beta$ cells and was 
associated with morphological changes and altered islet cell function, suggesting a direct cytopathic effect [39].

\section{What are T2DM screening procedures for HCV-infected patients?}

The American Diabetes Association (ADA) recommends diabetes testing for all patients 45 years or older, and in adults of any age who are overweight or obese $\left(B M I \geq 25 \mathrm{~kg} / \mathrm{m}^{2}\right.$, or $\geq 23 \mathrm{~kg} / \mathrm{m}^{2}$ in Asian Americans) who have one or more additional risk factor for diabetes [40]. Due to the increased risk of T2DM in HCV-infected patients, it has been proposed that all HCV-infected individuals be tested for T2DM [41], and T2DM testing in HCV-infected patients with cirrhosis is of particular importance. For diabetes testing the ADA recommends fasting plasma glucose, 2-hour plasma glucose during $75 \mathrm{~g}$ oral glucose tolerance test and A1C criteria as equally appropriate measures [40]. In cases of a normal test result, the ADA recommends retesting for T2DM every 3 years [40], but more frequent testing, either annually or bi-annually, may be beneficial to HCV-infected patients [41].

\section{How should patients with HCV-associated T2DM be managed?}

Consistent with international guidelines on the treatment of chronic HCV infection, including both EASL and AASLD treatment guidelines, HCV treatment should be initiated without delay for patients with clinically significant extrahepatic manifestations of chronic HCV, including those with T2DM [42-44]. As

well as treatment of $\mathrm{HCV}$, patients should also receive the appropriate standard of care for IR or T2DM, including dietary and lifestyle advice as well as appropriate diabetic medications and/or statins $[29,45]$.

Use of certain medications may protect from HCC in patients with chronic HCV infection and diabetes mellitus. The results of a systematic review suggest that use of metformin, sulfonylureas and insulin 
reduce the risk of HCC in patients with diabetes mellitus [46]. Two of the 10 studies included in the meta-analysis included only patients with chronic HCV infection and cirrhosis. In one of these studies [47], a multivariate analysis showed that use of metformin was independently associated with a marked reduction in the incidence of $\mathrm{HCC}$ over 5 years of follow-up (HR: $0.19 ; 95 \% \mathrm{Cl}: 0.04-0.79 ; \mathrm{P}=0.023$ ). Consistent with these results, an analysis of a large data base showed that male sex, cirrhosis, and chronic infection with HBV or HCV independently predicted development of HCC in patients with diabetes mellitus [48]. In particular the presence of diabetes mellitus, cirrhosis and chronic HCV infection was associated with an HR of 72.4 (95\% Cl 42.9-122) for development of HCC, Importantly use of metformin was associated with a reduced risk of HCC in this analysis. Continued use of metformin has also been shown to be associated with improved survival in patients who were using metformin at the time that cirrhosis is diagnosed (HR: $0.43 ; 95 \% \mathrm{Cl}: 0.24-0.78 ; \mathrm{P}=0.005)$ [49]. It should be noted, however, that few patients had HCV-associated cirrhosis in the analysis by Zhang et al [49].

Statins may protect against development of cancer including HCC. The use of statins has also been shown to protect against the development of HCC in a dose-related manner in patients with newly diagnosed T2DM [50]. More specifically, use of statins by patients with chronic HCV infection has been shown to be associated with a significantly lower incidence of HCC [51].

It is important that clinicians recognise HCV-associated T2DM and other extrahepatic manifestations of chronic HCV infection so that patients can receive timely diagnosis and treatment.

\section{What impact does HCV-associated T2DM have on efficacy of HCV treatment?}


Three meta-analyses, each assessing over $2100 \mathrm{HCV}$-infected patients, reported that patients with IR were less likely to achieve sustained virologic response (SVR) with interferon (IFN)-based HCV therapy than patients without IR [52-54]. In addition, two large cohort studies of HCV-infected patients who received IFN-based HCV therapy, one comprised of HCV GT1-3-infected US Veterans [55], and the other of Taiwanese patients [56], found that diabetes negatively impacted SVR rates. In the study of US Veterans, a traditionally difficult-to-treat population, the SVR rate in diabetic patients was $20 \%$ $(242 / 1,221)$ versus $29 \%(1,309 / 4,723)$ in non-diabetic patients $(P<0.001)$ [55]. In the Taiwanese study, which consisted of approximately half non-GT1 patients, the SVR rate in diabetic patients was 63\% $(160 / 253)$ versus $71 \%(867 / 1217)$ in non-diabetic patients $(P=0.008)[56]$.

The introduction of IFN-free direct-acting antiviral (DAA) HCV therapies has dramatically improved at SVR post-treatment week 12 (SVR12) rates in both clinical trials and real-world studies [57], and realworld study data suggest high efficacy in patients with T2DM [58-60]. In a cohort study of $4365 \mathrm{HCV}$ GT1-infected, treatment-naive US Veterans treated with ledipasvir and sofosbuvir with or without ribavirin (RBV), diabetes status did not impact SVR rates [58]. Among patients treated with ledipasvir and sofosbuvir without RBV, the SVR rate in patients with T2DM was 91\% $(955 / 1,047)$ versus $91 \%$ $(2,236 / 2,448)$ in those without diabetes [58]. Another real-world cohort study of $11,464 \mathrm{HCV}$-infected US Veterans treated with the DAA regimens of ledipasvir and sofosbuvir, of ombitasvir/paritaprevir/ritonavir and dasabuvir, or of simeprevir and sofosbuvir found no association between diabetes and reduced SVR rates [59]. Similarly, an observational cohort study of HCV GT1infected patients in Israel treated with ombitasvir/paritaprevir/ritonavir and dasabuvir found no link between diabetes and a reduction in SVR rate [60].

\section{What impact does treatment of HCV have on insulin resistance?}


There is evidence that suggests HCV eradication with IFN-based therapy has a positive effect on the risk for IR [61]. An Italian study evaluated non-diabetic GT1-4 HCV-infected patients treated with pegylated IFN + RBV and assessed the development of IR, defined as Homeostasis Model Assessment of Insulin Resistance (HOMA-IR) score <2 at baseline and then $>2$ at 24 months after HCV treatment. Here, 55\% (21/38) of patients with de novo IR had experienced treatment failure while $33 \%$ (103/316) of non-IR patients had treatment failure $(P=0.007)$ [61]. Additionally, SVR has been shown to improve IR in some HCV-infected patients. Among 300 HCV GT1-infected patients who achieved an SVR with interferonbased therapy, the prevalence of IR (HOMA-IR >3) decreased from $29 \%$ at baseline to $19 \% 12$ weeks after treatment $(P<0.001)$, and SVR was associated with a significant decrease in mean HOMA-IR over that time $(P=0.004)$ [62]. By contrast there was no significant change among HCV GT1-infected patients who failed treatment ( $n=197)$ [62]. A prospective study published in 2017, assessed the impact of HCV clearance with DAA treatment on IR and glycaemic control [63]. Patients ( $N=133)$ with HCV GT1infection and advanced liver fibrosis (F3-F4) without T2DM were included, with $n=68$ treated with DAAs and $n=65$ untreated. Baseline HOMA-IR were similar between groups. Patients who had HCV DAA therapy, had a significant reduction in HOMA-IR by the end of treatment ( $4.90 \pm 4.62$ baseline vs $2.42 \pm$ 1.85 at treatment end; $P<0.001$ ). Overall, $76.5 \%$ of patients who achieved a SVR had an improved IR and 41.2\% had a normalized IR. No improvement occurred in the untreated group. In patients with the highest fibrosis score, a degree of IR remained despite treatment [63].

\section{What impact does treatment of HCV have on the risk of de novo HCV-associated T2DM?}

HCV eradication with IFN-based therapy has been shown to reduce the risk for de novo T2DM [64-66]. A study of 1,059 HCV-infected patients, with or without T2DM, treated with IFN + RBV found that patients with a SVR were less likely to develop impaired fasting glucose or T2DM than those without (OR: 0.44; 
95\% $\mathrm{Cl} 0.20-0.97 ; P=0.004$ ) over a mean 27 months of follow-up [64]. In addition, A large Japanese retrospective cohort study of 2,842 patients with chronic HCV infection who were treated with IFNbased therapies, with a mean follow-up period of 6.4 years, demonstrated that patients who achieved an SVR12 had a 66\% reduction in the risk for T2DM (Figure 3) versus non-SVR patients and that this reduction was independent of age, presence of cirrhosis and pre-diabetes before therapy [65]. Similarly, an analysis of data from the US Veterans Affairs cohort (median follow-up of 3.2 years) showed that, despite poor overall SVR rates with pegylated IFN and RBV (31\%; 1,296/4,137), achievement of an SVR significantly reduced the likelihood of developing T2DM over time when compared to patients without an SVR (HR: $0.60 ; 95 \% \mathrm{Cl} 0.48-0.74 ; P<0.001)$ [66]. A real world analysis of 5,127 non-diabetic HCVinfected US patients, of whom $44 \%$ were treated with DAAs, found that over a mean follow-up of 3.7 years, patients who achieved an SVR had a $21 \%$ reduced risk of T2DM compared to patients who did not achieve an SVR (adjusted HR: 0.79; 95\% Cl: $0.65-0.96 ; P=0.02$ ), and the effect was consistent across age, race, BMI score and degree of fibrosis [67].

\section{What is the impact of treatment of HCV on established T2DM?}

There are limited data on the effect of viral eradication with DAAs on established T2DM, but initial studies of DAAs have shown beneficial metabolic changes in treated patients [68-70]. Significant reductions in glycated haemoglobin $\mathrm{A} 1 \mathrm{C}(\mathrm{HbA} 1 \mathrm{C})$, a measure of plasma glucose levels, have been shown in both non-diabetic and diabetic HCV-infected patients treated with DAAs $[68,69]$. A study of HCV GT1infected patients treated with sofosbuvir and RBV, the majority of whom were neither diabetic nor prediabetic, observed a significant decrease in $\mathrm{HbA} 1 \mathrm{C}$ from $5.58 \% \pm 0.08$ at baseline to $5.45 \% \pm 0.91$ at 24 weeks after end of treatment $(P=0.0046)$ [68]. Similarly, in a study of 60 HCV GT1-3-infected patients, with or without T2DM, treated with sofosbuvir-based therapy, a significant reduction in HbA1C was 
observed up to 6 months after treatment (Figure 4) [69]. The decrease in HbA1C was numerically greater in diabetic patients than non-diabetic patients, though the difference did not meet statistical significance. Furthermore, a post hoc analysis of Phase III trial data from HCV GT1-infected patients, with or without T2DM, who received ombitasvir/paritaprevir/ritonavir and dasabuvir with or without RBV or placebo for 12 weeks, found a greater decrease in adjusted mean glucose level at end of treatment in the DAA-treated patients versus those that received placebo $(-12.1 \mathrm{mg} / \mathrm{dL}$ for DAA-treated patients versus $-4.6 \mathrm{mg} / \mathrm{dL}$ for placebo-treated patients) [70]. In a second part of the post hoc analysis, long-term follow-up data from patients who received ombitasvir/paritaprevir/ritonavir and dasabuvir with or without RBV for 12 or 24 weeks were evaluated. In this evaluation, patients with diabetes (defined as patients with baseline fasting glucose value $>126 \mathrm{mg} / \mathrm{dL}$ ) or prediabetes (defined as patients with baseline fasting glucose value $100-126 \mathrm{mg} / \mathrm{dL}$ ) experienced on-treatment decreases in glucose levels that persisted up to 52 weeks post-treatment follow-up, while there was no significant change in glucose levels among non-diabetic patients (Figure 5) [70].

Similar results have been observed in real-world studies limited to T2DM patients treated with DAAs [71-73]. A small Italian retrospective study of chronic HCV-infected patients from two treatment centres identified 29 patients with T2DM who had been treated with DAAs (HCV GT1-4-infected patients) [71]. Using values for treatment weeks 4, 8 and/or 12, mean fasting glucose and $\mathrm{HbA} 1 \mathrm{C}$ levels were significantly lower versus baseline, and $84 \%(21 / 25)$ of patients experienced one or more of the following metabolic improvement criteria: a minimum $20 \mathrm{mg} / \mathrm{dL}$ decrease in fasting glucose level, a minimum $0.5 \%$ decrease in $\mathrm{HbA1C}$; or a reduction in dosage of hypoglycaemic drugs during $\mathrm{HCV}$ treatment [71]. Two Egyptian studies assessed the impact of sofosbuvir-based regimens on glycaemic control in predominantly HCV GT4-infected patients. One was a retrospective study of chronic HCVinfected patients with diabetes who achieved SVR24 with sofosbuvir-based therapy ( $n=65)$, and median fasting plasma glucose decreased from $113 \mathrm{mg} / \mathrm{dL}$ to $103 \mathrm{mg} / \mathrm{dL}(P=0.005)$ and median HbA1C level 
decreased from $6.9 \%$ to $6.4 \%(P<0.001)$, from baseline to SVR at post-treatment week 24 (SVR24), respectively [72]. A total of 51 (78\%) individuals experienced improvement in $\mathrm{HbA} 1 \mathrm{C}$ level (defined as a $0.5 \%$ decrease of from baseline level) at SVR24; lower BMI was associated with HbA1C decrease $(P=0.04)$ [72]. The second study assessed 460 Egyptian patients with chronic HCV GT4 infection with T2DM, and showed that treatment with sofosbuvir and daclatasvir, with or without RBV, was associated with improved glycaemic control in most patients [73]. Among 378 patients who achieved an SVR, 292 individuals (77\%) had improved glycaemic control [73]. Factors associated with improved glycaemic control identified by multivariate analysis were a duration of T2DM of less than 7 years, a negative family history of T2DM, and less severe cirrhosis (by Child-Pugh scale) [73].

Consistent with results from studies on IFN-based therapy, recent studies on DAA therapy have demonstrated the positive effects of viral eradication on T2DM $[74,75]$. An Italian study of HCV GT1-4infected patients with T2DM compared mean change in fasting glucose and $\mathrm{HbA1C}$ levels among patients who achieved SVR12 with DAA therapy to those who failed treatment or were untreated [74]. Patients who achieved SVR12 ( $n=101)$ experienced significant decreases in mean glucose, HbA1C, and HOMA-IR levels at post-treatment week 12 , while no significant changes in these variables were observed in those who failed treatment or were untreated $(n=21)$ [74]. In multivariable analysis, SVR12 was the only factor associated with improved glycaemic control (OR: 49.8; 95\% Cl: $2.2-112.7 ; P=0.014$ ) [74]. Additionally, an analysis of 2,435 US Veterans with T2DM and infected with HCV GT1 or GT4 who were treated with a DAA regimen, excluding RBV-containing regimens, assessed mean changes in HbA1C level before treatment and 15 months after treatment [75]. Among all treated patients, mean decrease in $\mathrm{HbA} 1 \mathrm{C}$ level was greater among those who achieved an SVR than those who failed treatment (mean difference $-0.18 \%$; $P=0.03$ ); when the analysis was limited to those with elevated $\mathrm{HbA} 1 \mathrm{C}$ at baseline (pretreatment $>7.2 \%)$, the mean difference was $-0.33 \%(P=0.02)(-0.34 \%[P=0.02]$ when adjusted for baseline factors) [75]. 
Two of the above studied assessed the impact of HCV therapy on antidiabetic medication use. In the Egyptian study of GT4 patients treated with sofosbuvir and daclatasvir, with or without RBV, among the 292 patients with improved glycaemic control, 78 (27\%) were able to decrease the dose of antidiabetic medication, either insulin ( $n=61)$ or gliclazide $(n=17)$ [73]. In the study of 2,435 HCV-infected US Veterans with T2DM treated with DAAs, achieving SVR was associated with decreased use of insulin; among patients who achieved SVR insulin use decreased from $41.3 \%$ before treatment to $38.0 \%$ after treatment, while it increased from $49.8 \%$ to $51.1 \%$ in non-SVR patients (mean difference between groups $=-4.5 \% ; P=0.04)[75]$.

\section{What is the impact of treatment of HCV on diabetic complications?}

Antiviral treatment with IFN-based therapy has been shown to lower the risk of extrahepatic manifestations of HCV infection, such as ESRD, acute coronary syndrome (ACS) and stroke [76,77], and viral clearance decreased the risk of chronic kidney disease and hemorrhagic stroke $[78,79]$. A large Taiwanese population-based study involving 2,822 patients with chronic HCV infection that was limited to those with diabetes mellitus demonstrated that treatment with pegylated IFN and RBV, without accounting for treatment response, was associated with reduced risk of diabetic complications such as ESRD, ischemic stroke and ACS [80]. Multivariate analyses demonstrated that among HCV-infected individuals with T2DM, antiviral treatment was associated with an $84 \%$ reduction in risk of ESRD (adjusted HR: 0.16; 95\% Cl: 0.07-0.33), a 47\% reduction in ischemic stroke (adjusted HR: 0.53; 95\% Cl: 0.30-0.93), and a 36\% reduction in ACS (adjusted HR: 0.64 ; $95 \% \mathrm{Cl}: 0.39-1.06$ ) over 8 years of follow-up [80].

Achievement of SVR in patients with bridging fibrosis or cirrhosis is associated with a greatly reduced incidence of HCC and liver related complications [81,82]; however, the risk of HCC is not completely 
eliminated after achievement of an SVR [83]. Until recently, conclusions regarding the impact of SVR on HCC incidence and other liver related outcomes have been based on studies from the interferon treatment era $[82,83]$. However, an analysis of a large US health system database has demonstrated that achievement of an SVR is associated with a reduced incidence of HCC regardless of whether a patient has received IFN-based treatment alone (HR: $0.32 ; 95 \% \mathrm{CI}: 0.28-0.37$ ), DAA plus IFN treatment (HR: $0.48 ; 95 \% \mathrm{Cl}: 0.32-0.73$ ) or DAA-based treatment alone (HR: $0.29 ; 95 \% \mathrm{Cl}: 0.23-0.37$ ) [84]. A subsequent prospective analysis of data from 2249 recipients of DAA-based regimens showed that the incidence of HCC was significantly lower in patients with compensated or decompensated cirrhosis who achieve an SVR $(P<0.001)$ [85]. Notwithstanding these observations, advanced age, higher platelet count and diabetes mellitus are associated with a higher risk of HCC in patients who have achieved an SVR [83]; thus, patient with cirrhosis and diabetes who achieve an SVR should be considered to be at increased risk of HCC than patients without diabetes.

These studies show the promise of DAA-based HCV therapy for patients with T2DM. In the daily care of HCV-infected T2DM patients treated with DAAs, close follow up is advisable to assess for changes in the patient's diabetes control status, including reduction in blood glucose, occurring either on-treatment or after stopping DAAs. This may necessitate a reduction of antidiabetic drugs. Additional studies on the effects of DAA therapy on risk of T2DM and associated complications, as well as on the subsequent management of T2DM are needed.

\section{Conclusions}

Multiple large meta-analyses have shown that chronic HCV infection is associated with an increased risk of T2DM $[7,8]$. There is strong evidence that T2DM in the context of HCV infection results in increased risk of adverse liver outcomes, such as advanced fibrosis, decompensated cirrhosis and HCC [15-24]. While T2DM has been found to reduce the efficacy of interferon-based HCV therapy, early studies 
suggest T2DM does not have the same effect on interferon-free DAA-based therapy [58-60].

Extrahepatic benefits of viral eradication in HCV-infected patients, including reduced risk of new-onset T2DM and reduced risk of diabetic complications in those already with the disease, underscore the need to evaluate all persons with HCV infection for diabetes and to treat HCV infection regardless of level of hepatic fibrosis. Heightened awareness of the vulnerability of diabetic patients with HCV to more advanced hepatic fibrosis and the need to prevent and manage complications of their liver disease is needed.

\section{Acknowledgement}

Medical editing support was provided by Scott Battle, Blair Jarvis and Gillian Patman of Medical Expressions, funded by AbbVie.

\section{Conflict of Interests:}

Luigi Elio Adinolfi: No conflicts of interest to report

Ira Jacobson: Research support: Gilead, Merck; Consultant/Advisor: AbbVie, BMS, Gilead, Janssen, Merck, Trek; Speaker: AbbVie, Gilead, Intercept, Merck

Mark Bondin: Employee of AbbVie and may hold stock or options.

Patrice Cacoub: Received consulting and lecturing fees from Abbvie, Astra Zeneca, Bristol-Myers Squibb, Gilead, Glaxo Smith Kline, Janssen, Merck Sharp Dohme, Roche, Servier and Vifor; received grants from CNRS, INSERM, Université Pierre et Marie Curie, ANRS and WHO. 


\section{References}

(1) Polaris Observatory HCV Collaborators. Global prevalence and genotype distribution of hepatitis C virus infection in 2015: a modelling study. Lancet Gastroenterol Hepatol. 2017;2:161-176.

(2) Gower E, Estes C, Blach S, Razavi-Shearer K, Razavi H. Global epidemiology and genotype distribution of the hepatitis C virus infection. J Hepatol. 2014;61:S45-57.

(3) Sebastiani G, Gkouvatsos K, Pantopoulos K. Chronic hepatitis C and liver fibrosis. World J Gastroenterol. 2014;20:11033-11053.

(4) Cacoub P, Gragnani L, Comarmond C, Zignego AL. Extrahepatic manifestations of chronic hepatitis C virus infection. Dig Liver Dis. 2014;46 Suppl 5:S165-73.

(5) Desbois AC, Cacoub P. Diabetes mellitus, insulin resistance and hepatitis C virus infection: A contemporary review. World J Gastroenterol. 2017;23:1697-1711.

(6) Shiffman ML, Gunn NT. Impact of hepatitis C virus therapy on metabolism and public health. Liver Int. 2017;37 Suppl 1:13-18.

(7) Younossi Z, Park H, Henry L, Adeyemi A, Stepanova M. Extrahepatic Manifestations of Hepatitis C: A Meta-analysis of Prevalence, Quality of Life, and Economic Burden. Gastroenterology. 2016;150:15991608.

(8) White DL, Ratziu V, El-Serag HB. Hepatitis C infection and risk of diabetes: a systematic review and meta-analysis. J Hepatol. 2008;49:831-844.

(9) Antonelli A, Ferri C, Fallahi P, et al. Hepatitis C virus infection: evidence for an association with type 2 diabetes. Diabetes Care. 2005;28:2548-2550.

(10) Knobler H, Schihmanter R, Zifroni A, Fenakel G, Schattner A. Increased risk of type 2 diabetes in noncirrhotic patients with chronic hepatitis C virus infection. Mayo Clin Proc. 2000;75:355-359.

(11) Mason AL, Lau JY, Hoang N, et al. Association of diabetes mellitus and chronic hepatitis C virus infection. Hepatology. 1999;29:328-333.

(12) Zein NN, Abdulkarim AS, Wiesner RH, Egan KS, Persing DH. Prevalence of diabetes mellitus in patients with end-stage liver cirrhosis due to hepatitis $\mathrm{C}$, alcohol, or cholestatic disease. J Hepatol. 2000;32:209-217.

(13) Hui JM, Sud A, Farrell GC, et al. Insulin resistance is associated with chronic hepatitis C virus infection and fibrosis progression [corrected. Gastroenterology. 2003;125:1695-1704.

(14) Guo X, Jin M, Yang M, Liu K, Li JW. Type 2 diabetes mellitus and the risk of hepatitis C virus infection: a systematic review. Sci Rep. 2013;3:2981. 
(15) Leandro G, Mangia A, Hui J, et al. Relationship between steatosis, inflammation, and fibrosis in chronic hepatitis C: a meta-analysis of individual patient data. Gastroenterology. 2006;130:1636-1642.

(16) Petta S, Camma C, Di Marco V, et al. Insulin resistance and diabetes increase fibrosis in the liver of patients with genotype $1 \mathrm{HCV}$ infection. Am J Gastroenterol. 2008;103:1136-1144.

(17) Miyaaki H, Ichikawa T, Taura N, et al. Predictive value of the fibrosis scores in patients with chronic hepatitis C associated with liver fibrosis and metabolic syndrome. Intern Med. 2011;50:1137-1141.

(18) Persico M, Masarone M, La Mura V, et al. Clinical expression of insulin resistance in hepatitis $C$ and B virus-related chronic hepatitis: differences and similarities. World J Gastroenterol. 2009;15:462-466.

(19) Li X, Gao Y, Xu H, Hou J, Gao P. Diabetes mellitus is a significant risk factor for the development of liver cirrhosis in chronic hepatitis C patients. Sci Rep. 2017;7:9087-017-09825-7.

(20) Huang YW, Yang SS, Fu SC, et al. Increased risk of cirrhosis and its decompensation in chronic hepatitis C patients with new-onset diabetes: a nationwide cohort study. Hepatology. 2014;60:807-814.

(21) Liu TL, Trogdon J, Weinberger M, Fried B, Barritt AS,4th. Diabetes Is Associated with Clinical Decompensation Events in Patients with Cirrhosis. Dig Dis Sci. 2016;61:3335-3345.

(22) Li X, Xu H, Gao Y, Pan M, Wang L, Gao P. Diabetes mellitus increases the risk of hepatocellular carcinoma in treatment-naive chronic hepatitis $\mathrm{C}$ patients in China. Medicine (Baltimore). 2017;96:e6508.

(23) Huang YW, Wang TC, Yang SS, et al. Increased risk of hepatocellular carcinoma in chronic hepatitis C patients with new onset diabetes: a nation-wide cohort study. Aliment Pharmacol Ther. 2015;42:902911.

(24) Veldt BJ, Chen W, Heathcote EJ, et al. Increased risk of hepatocellular carcinoma among patients with hepatitis C cirrhosis and diabetes mellitus. Hepatology. 2008;47:1856-1862.

(25) Rueger S, Bochud PY, Dufour JF, et al. Impact of common risk factors of fibrosis progression in chronic hepatitis C. Gut. 2015;64:1605-1615.

(26) Elkrief L, Chouinard P, Bendersky N, et al. Diabetes mellitus is an independent prognostic factor for major liver-related outcomes in patients with cirrhosis and chronic hepatitis C. Hepatology.

2014;60:823-831.

(27) Gregg EW, Li Y, Wang J, et al. Changes in diabetes-related complications in the United States, 19902010. N Engl J Med. 2014;370:1514-1523.

(28) Dzau VJ, Antman EM, Black HR, et al. The cardiovascular disease continuum validated: clinical evidence of improved patient outcomes: part I: Pathophysiology and clinical trial evidence (risk factors through stable coronary artery disease). Circulation. 2006;114:2850-2870. 
(29) Negro F. Facts and fictions of HCV and comorbidities: steatosis, diabetes mellitus, and cardiovascular diseases. J Hepatol. 2014;61:S69-78.

(30) Kralj D, Virovic Jukic L, Stojsavljevic S, Duvnjak M, Smolic M, Curcic IB. Hepatitis C Virus, Insulin Resistance, and Steatosis. J Clin Transl Hepatol. 2016;4:66-75.

(31) Aytug S, Reich D, Sapiro LE, Bernstein D, Begum N. Impaired IRS-1/PI3-kinase signaling in patients with HCV: a mechanism for increased prevalence of type 2 diabetes. Hepatology. 2003;38:1384-1392.

(32) Lerat $\mathrm{H}$, Imache $\mathrm{MR}$, Polyte J, et al. Hepatitis $\mathrm{C}$ virus induces a prediabetic state by directly impairing hepatic glucose metabolism in mice. J Biol Chem. 2017;292:12860-12873.

(33) Vanni E, Abate ML, Gentilcore E, et al. Sites and mechanisms of insulin resistance in nonobese, nondiabetic patients with chronic hepatitis C. Hepatology. 2009;50:697-706.

(34) Milner KL, van der Poorten D, Trenell M, et al. Chronic hepatitis C is associated with peripheral rather than hepatic insulin resistance. Gastroenterology. 2010;138:932-41.e1-3.

(35) Sabir SN, Manzoor S, Parvaiz F, Saalim M, Ghani E. Quantitative evaluation of insulin resistance markers in Pakistani patients suffering from HCV-associated type 2 diabetes mellitus. Acta Virol.

2017;61:197-203.

(36) Vanni E, Bugianesi E, Saracco G. Treatment of type 2 diabetes mellitus by viral eradication in chronic hepatitis C: Myth or reality? Dig Liver Dis. 2016;48:105-111.

(37) Greenberg AS, McDaniel ML. Identifying the links between obesity, insulin resistance and beta-cell function: potential role of adipocyte-derived cytokines in the pathogenesis of type 2 diabetes. Eur J Clin Invest. 2002;32 Suppl 3:24-34.

(38) Narita R, Abe S, Kihara Y, Akiyama T, Tabaru A, Otsuki M. Insulin resistance and insulin secretion in chronic hepatitis C virus infection. J Hepatol. 2004;41:132-138.

(39) Masini M, Campani D, Boggi U, et al. Hepatitis $C$ virus infection and human pancreatic beta-cell dysfunction. Diabetes Care. 2005;28:940-941.

(40) American Diabetes Association. 2. Classification and Diagnosis of Diabetes: Standards of Medical Care in Diabetes-2018. Diabetes Care. 2018;41:S13-S27.

(41) Hammerstad SS, Grock SF, Lee HJ, Hasham A, Sundaram N, Tomer Y. Diabetes and Hepatitis C: A Two-Way Association. Front Endocrinol (Lausanne). 2015;6:134.

(42) European Association for the Study of the Liver (EASL). EASL Recommendations on Treatment of Hepatitis C 2016. J Hepatol. 2017;66:153-194.

(43) AASLD and IDSA. HCV Guidance: Recommendations for testing, managing, and treating hepatitis C. 2017; Available at: http://www.hcvguidelines.org/. 
(44) Ramos-Casals M, Zignego AL, Ferri C, et al. Evidence-based recommendations on the management of extrahepatic manifestations of chronic hepatitis C virus infection. J Hepatol. 2017;66:1282-1299.

(45) Zignego AL, Ramos-Casals M, Ferri C, et al. International therapeutic guidelines for patients with HCV-related extrahepatic disorders. A multidisciplinary expert statement. Autoimmun Rev. 2017;16:523541.

(46) Singh S, Singh PP, Singh AG, Murad MH, Sanchez W. Anti-diabetic medications and the risk of hepatocellular cancer: a systematic review and meta-analysis. Am J Gastroenterol. 2013;108:881-91; quiz 892.

(47) Nkontchou G, Cosson E, Aout M, et al. Impact of metformin on the prognosis of cirrhosis induced by viral hepatitis C in diabetic patients. J Clin Endocrinol Metab. 2011;96:2601-2608.

(48) Lai SW, Chen PC, Liao KF, Muo CH, Lin CC, Sung FC. Risk of hepatocellular carcinoma in diabetic patients and risk reduction associated with anti-diabetic therapy: a population-based cohort study. Am J Gastroenterol. 2012;107:46-52.

(49) Zhang X, Harmsen WS, Mettler TA, et al. Continuation of metformin use after a diagnosis of cirrhosis significantly improves survival of patients with diabetes. Hepatology. 2014;60:2008-2016.

(50) Kim G, Jang SY, Han E, et al. Effect of statin on hepatocellular carcinoma in patients with type 2 diabetes: A nationwide nested case-control study. Int J Cancer. 2017;140:798-806.

(51) Tsan YT, Lee CH, Ho WC, Lin MH, Wang JD, Chen PC. Statins and the risk of hepatocellular carcinoma in patients with hepatitis C virus infection. J Clin Oncol. 2013;31:1514-1521.

(52) Deltenre $P$, Louvet $A$, Lemoine $M$, et al. Impact of insulin resistance on sustained response in HCV patients treated with pegylated interferon and ribavirin: a meta-analysis. J Hepatol. 2011;55:1187-1194.

(53) Eslam M, Aparcero R, Kawaguchi T, et al. Meta-analysis: insulin resistance and sustained virological response in hepatitis C. Aliment Pharmacol Ther. 2011;34:297-305.

(54) Laurito MP, Parise ER. Association between insulin resistance and sustained virologic response in hepatitis C treatment, genotypes 1 versus 2 and 3: systematic literature review and meta-analysis. Braz J Infect Dis. 2013;17:555-563.

(55) Backus LI, Boothroyd DB, Phillips BR, Mole LA. Predictors of response of US veterans to treatment for the hepatitis $C$ virus. Hepatology. 2007;46:37-47.

(56) Hung $\mathrm{CH}$, Lee $\mathrm{CM}$, Wang JH, et al. Impact of diabetes mellitus on incidence of hepatocellular carcinoma in chronic hepatitis $\mathrm{C}$ patients treated with interferon-based antiviral therapy. Int J Cancer. 2011;128:2344-2352.

(57) Manns MP, Buti M, Gane E, et al. Hepatitis C virus infection. Nat Rev Dis Primers. 2017;3:17006. 
(58) Backus LI, Belperio PS, Shahoumian TA, Loomis TP, Mole LA. Real-world effectiveness of ledipasvir/sofosbuvir in 4,365 treatment-naive, genotype 1 hepatitis C-infected patients. Hepatology. 2016;64:405-414.

(59) Fox DS, McGinnis JJ, Tonnu-Mihara IQ, McCombs JS. Comparative treatment effectiveness of direct acting antiviral regimens for hepatitis C: Data from the Veterans administration. J Gastroenterol Hepatol. 2017;32:1136-1142.

(60) Leventer-Roberts M, Hammerman A, Brufman I, et al. Effectiveness of dasabuvir/ombitasvir/paritaprevir/ritonavir for hepatitis $C$ virus in clinical practice: A population-based observational study. PLoS One. 2017;12:e0176858.

(61) Aghemo A, Prati GM, Rumi MG, et al. Sustained virological response prevents the development of insulin resistance in patients with chronic hepatitis C. Hepatology. 2012;56:1681-1687.

(62) Thompson AJ, Patel K, Chuang WL, et al. Viral clearance is associated with improved insulin resistance in genotype 1 chronic hepatitis $C$ but not genotype 2/3. Gut. 2012;61:128-134.

(63) Adinolfi LE, Nevola R, Guerrera B, et al. HCV clearance by direct-acting antiviral treatments reverses insulin resistance in chronic hepatitis C patients. J Gastroenterol Hepatol. 2017.

(64) Romero-Gomez M, Fernandez-Rodriguez CM, Andrade RJ, et al. Effect of sustained virological response to treatment on the incidence of abnormal glucose values in chronic hepatitis $\mathrm{C}$. J Hepatol. 2008;48:721-727.

(65) Arase Y, Suzuki F, Suzuki Y, et al. Sustained virological response reduces incidence of onset of type 2 diabetes in chronic hepatitis C. Hepatology. 2009;49:739-744.

(66) Pandya P, Pant C, Taylor R, Oni O. Impact of sustained virological response to chronic hepatitic C antiviral therapy on new onset diabetes mellitus type 2 after controlling for metabolic syndrome. J Investig Med. 2017;65:765-771.

(67) Li J, Zhang T, Gordon SC, et al. Impact of sustained virological response on incidence of type 2 diabetes in hepatitis C patients. Hepatology. 2017;66:531A.

(68) Meissner EG, Lee YJ, Osinusi A, et al. Effect of sofosbuvir and ribavirin treatment on peripheral and hepatic lipid metabolism in chronic hepatitis $C$ virus, genotype 1-infected patients. Hepatology.

2015;61:790-801.

(69) Morales AL, Junga Z, Singla MB, Sjogren M, Torres D. Hepatitis C eradication with sofosbuvir leads to significant metabolic changes. World J Hepatol. 2016;8:1557-1563.

(70) Mehta DA, Cohen E, Charafeddine M, et al. Effect of Hepatitis C Treatment with Ombitasvir/Paritaprevir/R + Dasabuvir on Renal, Cardiovascular and Metabolic Extrahepatic Manifestations: A Post-Hoc Analysis of Phase 3 Clinical Trials. Infect Dis Ther. 2017;6:515-529. 
(71) Pavone P, Tieghi T, d'Ettorre G, et al. Rapid decline of fasting glucose in HCV diabetic patients treated with direct-acting antiviral agents. Clin Microbiol Infect. 2016;22:462.e1-462.e3.

(72) Abdel Alem S, Elsharkawy A, Fouad R, et al. Improvement of glycemic state among responders to Sofosbuvir-based treatment regimens: Single center experience. J Med Virol. 2017;89:2181-2187.

(73) Dawood AA, Nooh MZ, Elgamal AA. Factors Associated with Improved Glycemic Control by DirectActing Antiviral Agent Treatment in Egyptian Type 2 Diabetes Mellitus Patients with Chronic Hepatitis C Genotype 4. Diabetes Metab J. 2017;41:316-321.

(74) Ciancio A, Bosio R, Bo S, et al. Significant improvement of glycemic control in diabetic patients with HCV infection responding to direct-acting antiviral agents. J Med Virol. 2018;90:320-327.

(75) Hum J, Jou JH, Green PK, et al. Improvement in Glycemic Control of Type 2 Diabetes After Successful Treatment of Hepatitis C Virus. Diabetes Care. 2017;40:1173-1180.

(76) Hsu YC, Ho HJ, Huang YT, et al. Association between antiviral treatment and extrahepatic outcomes in patients with hepatitis C virus infection. Gut. 2015;64:495-503.

(77) Hsu CS, Kao JH, Chao YC, et al. Interferon-based therapy reduces risk of stroke in chronic hepatitis C patients: a population-based cohort study in Taiwan. Aliment Pharmacol Ther. 2013;38:415-423.

(78) Arase Y, Suzuki F, Kawamura Y, et al. Development rate of chronic kidney disease in hepatitis C virus patients with advanced fibrosis after interferon therapy. Hepatol Res. 2011;41:946-954.

(79) Arase Y, Kobayashi M, Kawamura Y, et al. Impact of virus clearance for the development of hemorrhagic stroke in chronic hepatitis C. J Med Virol. 2014;86:169-175.

(80) Hsu YC, Lin JT, Ho HJ, et al. Antiviral treatment for hepatitis C virus infection is associated with improved renal and cardiovascular outcomes in diabetic patients. Hepatology. 2014;59:1293-1302.

(81) Bruno S, Stroffolini T, Colombo M, et al. Sustained virological response to interferon-alpha is associated with improved outcome in HCV-related cirrhosis: a retrospective study. Hepatology. 2007;45:579-587.

(82) van der Meer AJ, Veldt BJ, Feld JJ, et al. Association between sustained virological response and allcause mortality among patients with chronic hepatitis $C$ and advanced hepatic fibrosis. JAMA.

2012;308:2584-2593.

(83) van der Meer AJ, Feld JJ, Hofer H, et al. Risk of cirrhosis-related complications in patients with advanced fibrosis following hepatitis C virus eradication. J Hepatol. 2017;66:485-493.

(84) loannou GN, Green PK, Berry K. HCV eradication induced by direct-acting antiviral agents reduces the risk of hepatocellular carcinoma. J Hepatol. 2017.

(85) Calvaruso V, Cabibbo G, Cacciola I, et al. Incidence of Hepatocellular Carcinoma in Patients with HCV-associated Cirrhosis Treated with Direct-Acting Antiviral Agents. Gastroenterology. 2018. 
Figure 1. Cumulative incidence of cirrhosis in patients with chronic HCV infection, by presence of diabetes[20]

Figure 2: Kaplan-Meier curve showing cumulative incidence of HCC over time, by presence of diabetes and Ishak fibrosis score[24]

Figure 3. Cumulative incidence of type 2 diabetes mellitus following treatment with interferon-based therapies, by achievement of sustained virologic response at week 12[65]

Figure 4: Glycated hemoglobin levels in patients with chronic HCV infection before and after (up to 6 months) treatment with sofosbuvir-based therapy[69]

Figure 5: Predicted change in glucose level after treatment with ombitasvir/paritaprevir/ritonavir + dasabuvir with or without RBV among HCV-infected patients with baseline diabetes, pre-diabetes or without diabetes[70] 